\title{
AUTOMORPHISM GROUPS AND PICARD GROUPS OF ADDITIVE FULL SUBCATEGORIES
}

\author{
NAOYA HIRAMATSU and YUJI YOSHINO
}

\begin{abstract}
We study category equivalences between additive full subcategories of module categories over commutative rings. And we are able to define the Picard group of additive full subcategories. The aim of this paper is to study the properties of the Picard groups and show that the automorphism group of an additive full subcategory is a semi-direct product of the Picard group with the group of algebra automorphisms of the ring.
\end{abstract}

\section{Introduction}

Let $(A, \mathfrak{m})$ be a (commutative) Cohen-Macaulay local ring and $\mathrm{CM}(A)$ be the category consisting of maximal Cohen-Macaulay modules over $A$ and $A$ module homomorphisms. It is known (e.g. [12]) that the structure of $\mathrm{CM}(A)$ reflects the properties of the singularity of $\operatorname{Spec}(A)$. To understand $\operatorname{CM}(A)$ well, we consider in this paper the problem how big (or small) is the automorphism group of the category $\mathrm{CM}(A)$.

Our main theorem in the paper is the following (see Theorem 6.2).

Theorem 1.1. Let A be a Cohen-Macaulay local k-algebra with dimension $d$, where $k$ is a commutative ring. Suppose that A has only an isolated singularity (i.e., $A_{\mathfrak{p}}$ is a regular local ring for each prime ideal $\mathfrak{p}$ except $\mathrm{m}$ ). Let $\operatorname{Aut}_{k}(\mathrm{CM}(A))$ denote the group of $k$-linear automorphisms of the category $\mathrm{CM}(A)$. Then we have the following isomorphisms of groups.

$$
\operatorname{Aut}_{k}(\mathrm{CM}(A)) \cong \begin{cases}\operatorname{Aut}_{k \text {-alg }}(A) & (d \neq 2) \\ \operatorname{Aut}_{k \text {-alg }}(A) \ltimes C \ell(A) & (d=2),\end{cases}
$$

where $\operatorname{Aut}_{k \text {-alg }}(A)$ denotes the group of $k$-algebra automorphisms of $A$ and $C \ell(A)$ denotes the divisor class group of $A$.

Motivated by this result, we generalize in this paper these isomorphisms to hold for much wider classes of additive full subcategories 5 of $A$-Mod, and we shall show a certain structure theorem for $\operatorname{Aut}_{k}(\mathfrak{C})$ in the most generality.

Received 27 November 2008. 
Our primary setting is the following: Let $k$ be a commutative ring and $A$ be a commutative $k$-algebra. Assume that $\mathfrak{C}$ is an additive full subcategory of the module category over $A$ such that $\subseteq$ contains $A$ as an object. Since $A$ is a $k$-algebra, every additive full subcategory $\subseteq$ is a $k$-category. We say that a covariant functor $\mathfrak{C} \rightarrow \mathfrak{C}$ is a $k$-linear automorphism of $\mathfrak{C}$ if it is a $k$-linear functor giving an auto-equivalence of the category $\mathfrak{E}$. Then the set of all the isomorphism classes of $k$-linear automorphisms of $\mathfrak{E}$, which we denote by $\operatorname{Aut}_{k}(\mathfrak{S})$, forms a group by defining the multiplication to be the composition of functors. The final goal of this paper is to prove the isomorphism of groups

$$
\operatorname{Aut}_{k}(\mathfrak{S}) \cong \operatorname{Aut}_{k \text {-alg }}(A) \ltimes \operatorname{Pic}(\mathfrak{S}),
$$

under some subtle assumptions on (5. In this isomorphism, Pic(ङ) is the Picard group of 5 which we shall define in this paper below. Note that this type of isomorphism is known for the classical Picard group of the ring. (Compare with [2, Proposition 5.4, Chapter 2] and its non-commutative analogue in [11].) However, an original point of this paper is that we can define the Picard group for any additive full subcategory of module category over $A$ whenever it contains $A$ as an object.

In Section 2, we shall prove some results on the presentation of functors which give equivalences between additive full subcategories. See Theorems 2.5 and 2.7. These results can be regarded as part of a generalization of Morita theory. There are many variants and generalizations of Morita theory. See Rickard [9], [10] for other variants of Morita theory to derived categories. We should emphasize that the commutativity of the ring $A$ is essential in our argument in Section 2.

Based on the results in Section 2, we give the definition of the Picard group for an additive full subcategory in Section 3. See Definition 3.2.

In Section 4, we shall give our computational results for the Picard groups of various additive full subcategories. It is worth noting that if $A$ is a Krull domain and if $\mathfrak{C}$ is the category of reflexive $A$-lattices, then we shall have the

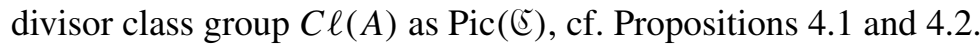

Section 5 is devoted to prove the above mentioned isomorphism (1) under the assumption that the category $\mathfrak{C}$ is stable under $\operatorname{Aut}_{k \text {-alg }}(A)$. See Definition 5.2 for the stability under $\operatorname{Aut}_{k \text {-alg }}(A)$. As a result, the Picard group $\operatorname{Pic}(\mathfrak{C})$ is naturally a normal subgroup $\operatorname{Aut}_{k}(\mathfrak{C})$ and the residue class group $\operatorname{Aut}_{k}\left(\mathfrak{C}^{5}\right) / \operatorname{Pic}\left(\mathfrak{C}^{5}\right)$ is isomorphic to $\operatorname{Aut}_{k \text {-alg }}(A)$, cf. Corollary 5.10.

In Section 6, we concentrate upon the category of Cohen-Macaulay modules over a Cohen-Macaulay local $k$-algebra. We prove the above mentioned Theorem 1.1 in Theorem 6.2. 
An application to the duality of an additive full subcategory is given in Section 7.

Convention. Throughout the paper, $k$ always denotes a commutative ring which we fix. Let $A$ be a commutative $k$-algebra. Then we denote the category of all $A$-modules and $A$-homomorphisms by $A$-Mod. When we say that $C$ is a subcategory of $A$-Mod, we always assume that $C$ is closed under isomorphisms, i.e., if $X$ and $Y$ are isomorphic $A$-modules and if $X$ is an object of $\mathbb{C}$, then so is $Y$. Since we only consider full subcategories of $A$-Mod in this paper, we often identify the full subcategory 5 of $A$-Mod with the class of objects of 5 . Hence we simply write $X \in \mathfrak{S}$ to indicate that $X$ is an object of $\mathfrak{5}$. A full subcategory C of $A$-Mod is said to be additive if it is closed under finite direct sums, i.e., if $X, Y \in \mathbb{C}$ then $X \oplus Y \in \mathbb{S}$.

\section{Equivalences of additive full subcategories}

Let $A$ and $B$ be commutative $k$-algebras and suppose we are given an additive full subcategory $\mathbb{C}$ (resp. $\mathfrak{D})$ of $A$-Mod (resp. $B$-Mod). And suppose that there is an additive covariant functor $F$ from 5 to $\mathfrak{D}$. We say that $F$ is a $k$-linear functor if it induces a $k$-linear mapping from $\operatorname{Hom}_{A}(X, Y)$ to $\operatorname{Hom}_{B}(F(X), F(Y))$ for any objects $X, Y \in \subseteq$. We denote the set of all the natural transformations $F \rightarrow F$ by $\operatorname{End}(F)$. Note that $\operatorname{End}(F)$ naturally has structure of abelian group, since $F: \mathfrak{C} \rightarrow \mathfrak{D}$ is an additive functor of additive categories. Moreover $\operatorname{End}(F)$ is a ring by defining the composition of natural transformations as the multiplication.

Since $A$ is a commutative ring, taking an element $a \in A$ we have the natural transformation $\alpha(a): F \rightarrow F$ defined by $\alpha(a)(X)=F\left(a_{X}\right): F(X) \rightarrow F(X)$ for each $X \in \mathbb{5}$, where $a_{X}$ denotes the multiplication map by $a$ on an object $X \in \mathfrak{C}^{5}$. (Note that $a_{X}: X \rightarrow X$ is an $A$-module homomorphism, since $A$ is a commutative ring.) In such a way we have the ring homomorphism

$$
\alpha: A \rightarrow \operatorname{End}(F) ; \quad a \mapsto F\left(a_{()}\right) .
$$

If $F$ is a $k$-linear functor, then it is easy to see that $\operatorname{End}(F)$ naturally has structure of $k$-algebra and that $\alpha$ is a $k$-algebra homomorphism.

Now suppose that $B \in \mathfrak{D}$ and there is an object $N \in \mathcal{C}$ such that $F(N) \cong B$. Note in this case that the mapping $B \rightarrow \operatorname{End}_{B}(F(N))$ which sends $b \in B$ to the multiplication mapping $b_{F(N)}$ by $b$ on $F(N)$ is an isomorphism of rings. Furthermore if $F$ is a $k$-linear functor, then this isomorphism is a $k$-algebra isomorphism. We often identify $\operatorname{End}_{B}(F(N))$ with $B$ by this isomorphism. Then we can define a ring homomorphism

$$
\beta: \operatorname{End}(F) \rightarrow \operatorname{End}_{B}(F(N)) \cong B ; \quad \varphi \mapsto \varphi(N) .
$$


If $F$ is a $k$-linear functor, then $\beta$ is a $k$-algebra homomorphism.

We observe the following lemma holds.

Lemma 2.1. Let $A$ and $B$ be commutative $k$-algebras and let $\mathbb{S}$ and $\mathfrak{D}$ be additive full subcategories of $A$-Mod and $B$-Mod respectively such that $A \in \mathbb{S}$ and $B \in \mathfrak{D}$. Then the following hold for a $k$-linear covariant functor $F:(5 \rightarrow$ D.

(1) If $F$ is a faithful functor, then the $k$-algebra homomorphism $\alpha$ is injective.

(2) If $F$ is a full functor and it has a right adjoint functor, then $\alpha$ is surjective.

(3) Assume that there is an object $N \in \mathbb{C}$ such that $F(N) \cong B$. Then the $k$-algebra homomorphism $\beta$ is surjective.

(4) Adding to the assumption of (3), if $F$ is a full functor, then $\beta$ is injective as well.

Proof. (1) Suppose $\alpha(a)=0$ for $a \in A$. Since $A$ is an object of 5 and since $F$ is a faithful functor, a natural mapping $\varphi_{A}: A=\operatorname{End}_{A}(A) \rightarrow$ $\operatorname{End}_{B}(F(A))$ defined by $\varphi_{A}(a)=F\left(a_{A}\right)$ is injective. Note that there is a commutative diagram

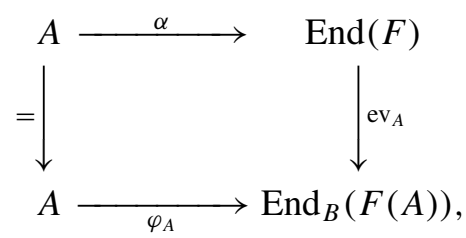

where the right vertical arrow $\operatorname{ev}_{A}$ is the evaluation map at $A$ which maps $\gamma \in \operatorname{End}(F)$ to $\gamma(A)$. This implies that $\varphi_{A}(a)=F\left(a_{A}\right)=\operatorname{ev}_{A}(\alpha(a))=0$, which forces $a=0$.

(2) Since $F$ is full, the natural map $\varphi_{A}: A=\operatorname{End}_{A}(A) \rightarrow \operatorname{End}_{B}(F(A))$ is surjective. If the mapping $\mathrm{ev}_{A}$ in the diagram of $(*)$ is injective, then it follows from the commutativity of $(*)$ that $\alpha$ is surjective. Thus we only have to show that $\mathrm{ev}_{A}$ is injective.

Let $G$ be a right adjoint functor of $F$ and we have a commutative diagram
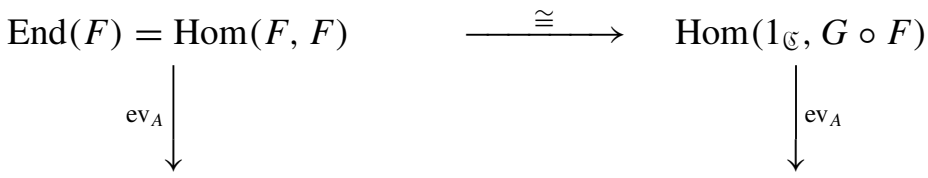

$\operatorname{End}_{B}(F(A))=\operatorname{Hom}_{B}(F(A), F(A)) \longrightarrow \operatorname{Hom}_{A}(A, G \circ F(A))$.

Therefore it is enough to show that $\mathrm{ev}_{A}: \operatorname{Hom}\left(1_{\mathfrak{E}}, G \circ F\right) \rightarrow \operatorname{Hom}_{A}(A, G \circ$ $F(A))$ is an injective map. 
To prove this, assume $\mathrm{ev}_{A}(\psi)=\psi(A)=0$ for $\psi \in \operatorname{Hom}\left(1_{\mathfrak{c}}, G \circ F\right)$. For any $X \in \mathcal{S}$ and any $x \in X$, we define an $A$-module homomorphism $h_{x}: A \rightarrow X$ by $h_{x}(a)=a x(a \in A)$. Since $\psi$ is a natural transformation from $1_{\mathfrak{c}}$ to $G \circ F$, there is a commutative diagram

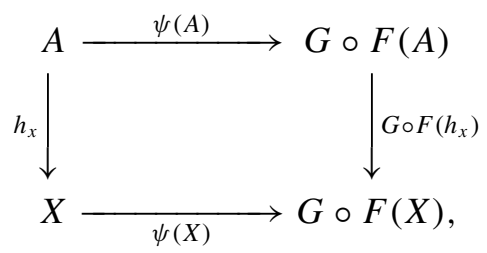

which implies that $\psi(X)(x)=0$ for all $x \in X$, since $\psi(A)=0$. Hence $\psi(X)=0$ for all $X \in \mathbb{E}$, and we conclude that $\psi=0$.

(3) For any $b \in B$ and any $X \in \mathcal{C}$, we denote by $\varphi_{b}(X)$ the multiplication map by $b$ on $F(X)$. Since $F$ is a functor, for any $f \in \operatorname{Hom}_{A}(X, Y)$ where $X, Y \in \mathfrak{C}, F(f)$ is a $B$-module homomorphism from $F(X)$ to $F(Y)$, therefore we have a commutative diagram

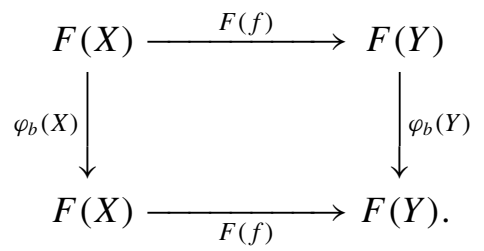

Thus $\varphi_{b}$ is a natural transformation of $F$, hence $\varphi_{b} \in \operatorname{End}(F)$ for all $b \in B$. Clearly the equality $\beta\left(\varphi_{b}\right)=b$ holds, hence $\beta$ is surjective.

(4) To prove the injectivity of $\beta$, let $\beta(\psi)=\psi(N)=0$ for $\psi \in \operatorname{End}(F)$. We have to show that $\psi(X): F(X) \rightarrow F(X)$ is a zero map for each $X \in \subseteq$. For any $y \in F(X)$, we define a $B$-module homomorphism $h_{y}: B \rightarrow F(X)$ by $h_{y}(b)=b y(b \in B)$. Since $F$ is a full functor and since $F(N) \cong B$, there exists an $f_{y} \in \operatorname{Hom}_{A}(N, X)$ such that $F\left(f_{y}\right)=h_{y}$. Then there is a commutative diagram

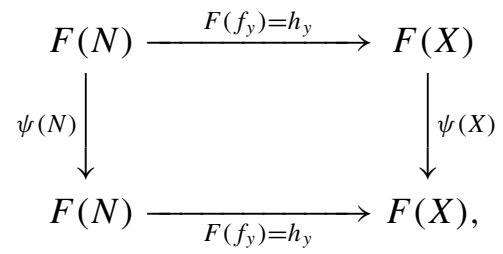

and hence it follows that $\psi(X)(y)=0$. Since $y$ is any element of $F(X)$, we have $\psi(X)=0$.

The lemma holds for a contravariant functor. Actually we can prove the following lemma whose proof will be left to the reader. 
Lemma 2.2. Let $A$ and $B$ be commutative $k$-algebras and let $\mathbb{S}$ and $\mathfrak{D}$ be additive full subcategories of $A$-Mod and $B$-Mod respectively such that $A \in \mathfrak{S}$ and $B \in \mathfrak{D}$. Then the following hold for a k-linear contravariant functor $F: \mathbb{C} \rightarrow \mathfrak{D}^{\mathrm{op}}$.

(1) If $F$ is a faithful functor, then the k-algebra homomorphism $\alpha$ is injective.

(2) If $F$ is a full functor and it has a right adjoint functor, then $\alpha$ is surjective.

(3) Assume that there is an object $N \in \mathfrak{C}$ such that $F(N) \cong B$. Then the $k$-algebra homomorphism $\beta$ is surjective.

(4) Adding to the assumption of (3), if $F$ is a full functor, then $\beta$ is injective as well.

Recall that we say that $\mathfrak{S}$ and $\mathfrak{D}$ are equivalent to each other as $k$-categories if there is a $k$-linear functor $\mathbb{C} \rightarrow \mathfrak{D}$ which gives an equivalence of categories.

Corollary 2.3. Let $A$ and $B$ be commutative k-algebras and let $\mathbb{5}$ and $D$ be additive full subcategories of $A-M o d$ and $B$-Mod respectively such that $A \in \mathfrak{C}$ and $B \in \mathfrak{D}$. Suppose the categories $\mathfrak{S}$ and $\mathfrak{D}$ are equivalent to each other as k-categories. Then $A$ is isomorphic to $B$ as a k-algebra.

Proof. This is a consequence of Lemma 2.1, since the $k$-linear equivalence $F: \mathfrak{C} \rightarrow \mathfrak{D}$ satisfies all the necessary conditions assumed in the lemma.

It is well known that there exist examples of non-commutative rings for which Corollary 2.3 does not hold. For example, let $A$ be a commutative ring and $B$ be an $m \times m$ full matrix algebra over $A$. Then, $A$ and $B$ are Morita equivalent, hence there is an equivalence of categories $A$-Mod $\cong B$-Mod. However, $A$ are $B$ are not isomorphic as algebras if $m \geqq 2$.

Next we argue about the form of a functor which gives an equivalence of categories.

Definition 2.4. Let $A$ and $B$ be commutative $k$-algebras and let $N$ be an $A$-module. Given a $k$-algebra homomorphism $\sigma: B \rightarrow A$, we define an $\left(A \otimes_{k} B\right.$ )-module $N_{\sigma}$ by $N_{\sigma}=N$ as an abelian group on which the ring action is defined by

$$
(a \otimes b) \cdot n=a \sigma(b) n
$$

for any $a \otimes b \in A \otimes_{k} B$ and $n \in N$. In such a case, we can define a functor

$$
\operatorname{Hom}_{A}\left(N_{\sigma},-\right): A-\operatorname{Mod} \rightarrow B \text {-Mod } .
$$

Note that the $B$-module structure on $\operatorname{Hom}_{A}\left(N_{\sigma}, X\right)$ for $X \in A$-Mod is defined by $(b \cdot f)(n)=f((1 \otimes b) \cdot n)$ for $f \in \operatorname{Hom}_{A}\left(N_{\sigma}, X\right), b \in B$ and $n \in N$. 
THEOREM 2.5. Let $A$ and $B$ be commutative $k$-algebras and let $\mathbb{E}$ and $\mathfrak{D}$ be additive full subcategories of $A-\operatorname{Mod}$ and $B$-Mod respectively such that $A \in \mathfrak{C}$ and $B \in \mathfrak{D}$. Suppose that $\mathfrak{C}$ and $\mathfrak{D}$ are equivalent as $k$-categories and let $F: \mathfrak{C} \rightarrow \mathfrak{D}$ be a $k$-linear covariant functor which gives an equivalence between them. Then there is a k-algebra isomorphism $\sigma: B \rightarrow A$ such that $F$ is isomorphic to the functor $\left.\operatorname{Hom}_{A}\left(N_{\sigma},-\right)\right|_{\mathfrak{S}}$, where $N \in \mathfrak{C}$ is chosen so that $F(N) \cong B$ in $\mathfrak{D}$.

Proof. Since $F$ is an equivalence, there exists an $A$-module $N \in \mathbb{C}$ such that $F(N) \cong B$. By virtue of Lemma 2.1, the $k$-algebra homomorphisms $\alpha$ and $\beta$ are defined well and they are isomorphisms. Now we define a $k$-algebra isomorphism $\sigma: B \rightarrow A$ as the composition of $\alpha^{-1}$ and $\beta^{-1}$ :

$$
\begin{aligned}
& B \stackrel{\cong}{\longrightarrow} \operatorname{End}_{B}(F(N)) \stackrel{\beta^{-1}}{\longrightarrow} \operatorname{End}(F) \stackrel{\alpha^{-1}}{\longrightarrow} A \\
& b \longrightarrow b_{F(N)} \longrightarrow \beta^{-1}\left(b_{F(N)}\right) \longrightarrow \sigma(b)=\alpha^{-1}\left(\beta^{-1}\left(b_{F(N)}\right)\right) .
\end{aligned}
$$

Note from the definition of $\alpha$ and $\beta$, the equality $F\left(\sigma(b)_{N}\right)=b_{F(N)}$ holds for any $b \in B$.

Since $F$ is a $k$-linear equivalence, we have isomorphisms of $k$-modules for each $X \in \mathfrak{c}$;

$$
\begin{aligned}
\operatorname{Hom}_{A}\left(N_{\sigma}, X\right) \stackrel{\cong}{\longrightarrow} \operatorname{Hom}_{B}(F(N), F(X)) \stackrel{\cong}{\longrightarrow} F(X) \\
f \longrightarrow F(f) \longrightarrow F(f)(1),
\end{aligned}
$$

whose composition we denote by $\varphi_{X}$. We claim that $\varphi_{X}$ is a $B$-module homomorphism for each $X \in \mathbb{E}$. For this, we show that $F(b \cdot f)=b F(f)$ for any $b \in B$ and any $f \in \operatorname{Hom}_{A}\left(N_{\sigma}, X\right)$. Since the equality $(b \cdot f)(n)=f((1 \otimes$ $b) \cdot n)=f(\sigma(b) n)=(\sigma(b) f)(n)=\sigma(b)_{X}(f(n))$ holds for any $n \in N$, we should note that $b \cdot f=\sigma(b)_{X} f$, hence we have $F(b \cdot f)=F\left(\sigma(b)_{X}\right) F(f)$. Therefore it is enough to show the equality $F\left(\sigma(b)_{X}\right)=b_{F(X)}$ for any $b \in B$ and $X \in \mathfrak{C}$. To prove this, for $y \in F(X)$, let $h_{y}: B \rightarrow F(X)$ be a $B$-module homomorphism defined by $h_{y}(b)=$ by $(b \in B)$. Since $F(N) \cong B$ and $F$ is full, there exists $g_{y} \in \operatorname{Hom}_{A}(N, X)$ such that $h_{y}=F\left(g_{y}\right)$. Since $g_{y}$ is an $A$-module homomorphism, we have the equality $\sigma(b)_{X} \cdot g_{y}=g_{y} \cdot \sigma(b)_{N}$ for $b \in B$. Thus we have $F\left(\sigma(b)_{X}\right) \cdot F\left(g_{y}\right)=F\left(g_{y}\right) \cdot F\left(\sigma(b)_{N}\right)$. This implies $F\left(\sigma(b)_{X}\right) \cdot h_{y}=h_{y} \cdot F\left(\sigma(b)_{N}\right)=h_{y} \cdot b_{F(N)}=b_{F(X)} \cdot h_{y}$, hence in particular, we have $F\left(\sigma(b)_{X}\right)(y)=b_{F(X)}(y)$ for all $y \in F(X)$. This shows that $F\left(\sigma(b)_{X}\right)=b_{F(X)}$ as desired.

As the final step of proof, we show that the $B$-module isomorphism $\varphi_{X}$ : $\operatorname{Hom}_{A}\left(N_{\sigma}, X\right) \rightarrow F(X)$ is functorial in the variable $X$ so that it induces the isomorphism of functors $\varphi: \operatorname{Hom}_{A}\left(N_{\sigma},-\right) \rightarrow F$. To prove this let $f:$ 
$X \rightarrow Y$ be any morphism in 5 . We want to show the equality $F(f) \cdot \varphi_{X}=$ $\varphi_{Y} \cdot \operatorname{Hom}_{A}\left(N_{\sigma}, f\right)$. But evaluating the both ends at $g \in \operatorname{Hom}_{A}\left(N_{\sigma}, X\right)$, we have $F(f)\left(\varphi_{X}(g)\right)=F(f)(F(g)(1))$, and $\left(\varphi_{Y} \cdot \operatorname{Hom}_{A}\left(N_{\sigma}, f\right)\right)(g)=\varphi_{Y}(f \cdot g)=$ $F(f \cdot g)(1)=F(f)(F(g)(1))$, hence the equality above holds.

It is worth noting the following corollary as a direct consequence of the theorem.

Corollary 2.6. Let $A$ and $B$ be commutative $k$-algebras and let $\mathfrak{C}$ and D be additive full subcategories of $A-\operatorname{Mod}$ and $B-\operatorname{Mod}$ respectively such that $A \in \mathfrak{C}$ and $B \in \mathfrak{D}$. Suppose that $F: \mathfrak{C} \rightarrow \mathfrak{D}$ is a $k$-linear covariant functor which gives an equivalence between them and that $F(A) \cong B$ in $\mathfrak{D}$. Then there is a k-algebra isomorphism $\sigma: B \rightarrow A$ such that $F$ is isomorphic to the functor $\left.\operatorname{Hom}_{A}\left(A_{\sigma},-\right)\right|_{\mathfrak{c}}$.

In particular, if $F: \mathfrak{S} \rightarrow \mathfrak{C}$ is a k-linear auto-equivalence of $\mathfrak{S}$ and if $F(A) \cong A$, then $F$ is isomorphic to the identity functor on $\mathfrak{S}$.

The contravariant version of the theorem also holds, whose proof will go through similarly to the proof of Theorem 2.5 , but using Lemma 2.2 instead of Lemma 2.1.

THeOREM 2.7. Let $A$ and $B$ be commutative $k$-algebras and let $\mathfrak{S}$ and $\mathfrak{D}$ be additive full subcategories of $A-\operatorname{Mod}$ and $B-\operatorname{Mod}$ respectively such that $A \in \mathfrak{C}$ and $B \in \mathfrak{D}$. Suppose there is a $k$-linear contravariant functor $F: \mathfrak{S} \rightarrow \mathfrak{D}^{\text {op }}$ which gives a duality between them. Then there is a k-algebra isomorphism $\sigma: B \rightarrow A$ such that $F$ is isomorphic to the functor $\left.\operatorname{Hom}_{A}\left(-, N_{\sigma}\right)\right|_{\mathfrak{c}}$ for $N \in \mathfrak{C}$ with $F(A) \cong B$.

Note that for any $X \in \mathfrak{C}$, the $B$-module structure on $\operatorname{Hom}_{A}\left(X, N_{\sigma}\right)$ is defined as follows:

$$
(b \cdot f)(x)=(1 \otimes b) \cdot f(x)=\sigma(b) f(x),
$$

for $x \in X, f \in \operatorname{Hom}_{A}\left(X, N_{\sigma}\right)$ and $b \in B$.

\section{Picard groups of additive full subcategories}

In this section, we study the group of all the $A$-linear automorphisms of an additive full subcategory of $A$-Mod.

Let $A$ be a commutative ring and let $\mathbb{S}$ be an additive full subcategory of $A$-Mod such that $A \in \mathfrak{C}$. We denote by $\operatorname{Aut}_{A}(\mathfrak{S})$ the group of all the isomorphism classes of $A$-linear automorphisms over $(5$, i.e.,

$$
\operatorname{Aut}_{A}(\mathfrak{S})=\left\{\begin{array}{l|l}
F: \mathfrak{S} \rightarrow \mathfrak{S} \mid \begin{array}{l}
F \text { is an } A \text {-linear covariant functor that } \\
\text { gives an equivalence of the category } \mathfrak{c}
\end{array}
\end{array}\right\} \cong .
$$


Since the identity mapping $A \rightarrow A$ is the unique $A$-algebra automorphism of $A$, Theorem 2.5 implies the following.

CoROllary 3.1. For any element $[F] \in \operatorname{Aut}_{A}(\mathfrak{C})$, there is an isomorphism of functors $\left.F \cong \operatorname{Hom}_{A}(N,-)\right|_{\Subset}$ for some $N \in \mathfrak{G}$.

Taking this corollary into consideration, we make the following definition.

Definition 3.2. Let $A$ be a commutative ring and let $\mathbb{C}$ be an additive full subcategory of $A$-Mod such that $A \in \mathbb{C}$. We define Pic(战) to be the set of all the isomorphism classes of $A$-modules $M \in \mathfrak{E}$ such that $\left.\operatorname{Hom}_{A}(M,-)\right|_{\mathfrak{E}}$ gives an equivalence of the category. That is,

$$
\begin{aligned}
& \operatorname{Pic}(\mathfrak{S})=\left\{M \in \mathfrak{S}\left|\operatorname{Hom}_{A}(M,-)\right|_{\mathfrak{E}}\right. \text { gives } \\
& \quad \text { an }(A \text {-linear }) \text { equivalence } \mathfrak{S} \rightarrow \mathfrak{S}\} / \cong .
\end{aligned}
$$

We define the group structure on $\operatorname{Pic}(\mathfrak{C})$ as follows: Let $[M]$ and $[N]$ be in $\operatorname{Pic}(\mathfrak{S})$. Since the composition $\left.\left.\operatorname{Hom}_{A}(M,-)\right|_{\mathfrak{E}} \circ \operatorname{Hom}_{A}(N,-)\right|_{\mathfrak{E}}$ is also an $A$-linear equivalence, it follows from Corollary 3.1 that there exists an $L \in \mathfrak{F}$ such that

$$
\left.\left.\left.\operatorname{Hom}_{A}(L,-)\right|_{\mathfrak{S}} \cong \operatorname{Hom}_{A}(M,-)\right|_{\mathfrak{⿷}} \circ \operatorname{Hom}_{A}(N,-)\right|_{\mathfrak{c}} .
$$

We define the multiplication in $\operatorname{Pic}(\complement)$ by $[M] \cdot[N]=[L]$. Note that

$$
\begin{aligned}
\left.\left.\operatorname{Hom}_{A}(M,-)\right|_{\Subset} \circ \operatorname{Hom}_{A}(N,-)\right|_{\Subset} & \left.\cong \operatorname{Hom}_{A}\left(M \otimes_{A} N,-\right)\right|_{\Subset} \\
& \left.\left.\cong \operatorname{Hom}_{A}(N,-)\right|_{\Subset} \circ \operatorname{Hom}_{A}(M,-)\right|_{\Subset},
\end{aligned}
$$

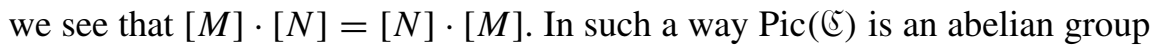
with the identity element $[A]$. We call Pic(C) the Picard group of $\mathfrak{C}^{5}$.

Note from Yoneda's lemma that objects $M$ and $N$ in $\subseteq$ are isomorphic as $A$-modules if and only if the functors $\left.\operatorname{Hom}_{A}(M,-)\right|_{\mathfrak{E}}$ and $\left.\operatorname{Hom}_{A}(N,-)\right|_{\mathfrak{E}}$ are isomorphic to each other. Therefore the multiplication in Pic(纤 is welldefined. We should note that the multiplication is not necessarily defined to be $M \otimes_{A} N$ in the usual way, since $M \otimes_{A} N$ is not always an object of $\mathfrak{C}$.

Furthermore, the mapping $\operatorname{Pic}(\mathfrak{C}) \rightarrow \operatorname{Aut}_{A}(\mathfrak{C})$ which sends $[M]$ to $\left.\operatorname{Hom}_{A}(M,-)\right|_{\mathfrak{E}}$ is an isomorphism of groups by Corollary 3.1.

Lemma 3.3. There is an isomorphism $\operatorname{Pic}(\mathfrak{S}) \cong \operatorname{Aut}_{A}(\mathfrak{S})$ as groups.

In the following we shall give some examples of the Picard groups of additive full subcategories. 
Let $A$ be a commutative ring. We denote by Pic $A$ the ordinary Picard group of the ring $A$. Recall that an $A$-module $M$ is called invertible if there is an $A$ module $M^{\prime}$ such that $M \otimes_{A} M^{\prime} \cong A$ as $A$-modules. Then Pic $A$ consists of all the isomorphism classes of invertible $A$-modules, and the multiplication in Pic $A$ is defined by tensor product over $A$. See [2, $\$ 5$, Chapter 2] for more details. Recall the following lemma holds for invertible modules. (Cf. [3].)

Lemma 3.4. Let $A$ be a commutative ring and let $M$ be an invertible Amodule. Then $M$ is a finitely generated projective A-module.

Adding to the lemma, we note that if $M$ is an invertible $A$-module, then there is an isomorphism $\operatorname{Hom}_{A}(M,-) \cong M^{*} \otimes_{A}-$ as functors on $A$-Mod, where $M^{*}$ denotes $\operatorname{Hom}_{A}(M, A)$. In fact, there is a natural transformation $M^{*} \otimes_{A}-\rightarrow \operatorname{Hom}_{A}(M,-)$ of functors that is an isomorphism if $M$ is a finitely generated projective $A$-module. Note also that $M \otimes_{A} M^{*} \cong A$ holds for an invertible $A$-module $M$.

Recall that an additive full subcategory is called additively closed if it is closed under summands of finite direct sums. Note that if $\mathfrak{C}$ is an additively closed full subcategory of $A$-Mod and if $A \in \widetilde{C}$, then all finitely generated projective $A$-modules belong to $\mathfrak{S}$, since they are summands of finite direct sums of copies of $A$. We note the following lemma holds.

Lemma 3.5. Let A be a commutative ring and let $\mathfrak{c}$ be a full subcategory of A-Mod that is additively closed and $A \in \mathfrak{E}$. Then the classes of invertible $A$-modules are elements of $\mathrm{Pic}(\mathfrak{S})$, and hence $\mathrm{Pic} A$ is naturally a subgroup of $\mathrm{Pic}(\mathfrak{C})$.

Proof. Let $[M] \in \operatorname{Pic} A$. Then $M \in \mathfrak{C}$ as remarked above. Assume that $M$ is a summand of the free module $A^{n}$ of rank $n$. Then, for any $X \in \mathfrak{C}$, $\operatorname{Hom}_{A}(M, X)$ is a summand of $\operatorname{Hom}_{A}\left(A^{n}, X\right) \cong X^{n}$ which is an object of ๔. Therefore we have $\operatorname{Hom}_{A}(M, X) \in \mathfrak{C}$ for any $X \in \mathfrak{C}$. This shows that $\operatorname{Hom}_{A}(M,-)$ defines a functor $\mathfrak{S} \rightarrow \mathfrak{C}$. Let $M^{\prime}$ be an $A$-module with $M \otimes_{A}$ $M^{\prime} \cong A$. Then by the same reason as above, $\operatorname{Hom}_{A}\left(M^{\prime},-\right)$ defines a functor $\mathfrak{5} \rightarrow \mathfrak{C}$ as well. Since $\left.\left.\operatorname{Hom}_{A}(M,-)\right|_{\mathfrak{c}} \circ \operatorname{Hom}_{A}\left(M^{\prime},-\right)\right|_{\mathfrak{C}} \cong \operatorname{Hom}_{A}\left(M \otimes_{A}\right.$ $\left.M^{\prime},-\right)\left.\right|_{\mathfrak{E}} \cong 1_{\mathfrak{E}}$, we see that $[M] \in \operatorname{Pic}(\mathfrak{C})$.

A full subcategory $\mathbb{E}$ of $A$-Mod is said to be closed under tensor products if $M \otimes_{A} N \in \mathfrak{C}$ for all $M, N \in \mathfrak{C}$.

Lemma 3.6. Let A be a commutative ring and let $\mathbb{5}$ be an additive full subcategory of $A-M o d$ that is closed under tensor products and $A \in \mathfrak{C}$. Then $\mathrm{Pic}(\mathfrak{S})$ is naturally a subgroup of Pic A.

Proof. Let $[M] \in \operatorname{Pic}(\mathfrak{C})$. Then there is an $M^{\prime} \in \mathfrak{C}$ such that $\operatorname{Hom}_{A}\left(M \otimes_{A}\right.$ 
$\left.M^{\prime},-\right)\left.\right|_{\mathfrak{E}} \cong 1_{\mathfrak{E}}$. Since $M \otimes_{A} M^{\prime} \in \mathfrak{C}$, Yoneda's lemma implies that $M \otimes_{A} M^{\prime} \cong$ $A$ as $A$-modules, hence $M$ is an invertible $A$-module. Therefore $[M] \in \operatorname{Pic} A$.

Combining Lemmas 3.5 and 3.6 above, we have the following proposition.

Proposition 3.7. Let A be a commutative ring and let $\subseteq$ be an additively closed full subcategory of A-Mod which is closed under tensor products and $A \in \mathbb{S}$. Then we have the equality

$$
\operatorname{Pic}(5)=\operatorname{Pic} A \text {. }
$$

Note in the proposition that the equality means an equality of subsets of the set of all the isomorphism classes of $A$-modules.

Example 3.8. Let $A$ be a commutative ring. We denote by $A$-mod the full subcategory of $A$-Mod consisting of all finitely generated $A$-modules. We also denote by $\operatorname{Proj}(A)($ resp. $\operatorname{proj}(A))$ the full subcategory of $A$-Mod consisting of all projective $A$-modules (resp. all finitely generated projective $A$-modules). Since all of these full subcategories are additively closed and closed under tensor products, we have the equalities

$$
\operatorname{Pic}(A-\operatorname{Mod})=\operatorname{Pic}(A-\bmod )=\operatorname{Pic}(\operatorname{Proj}(A))=\operatorname{Pic}(\operatorname{proj}(A))=\operatorname{Pic} A .
$$

\section{Further examples of Picard groups}

In this section we shall give numerous examples of Picard groups for additive subcategories.

Let $A$ be a Krull domain. We denote by $C \ell(A)$ the divisor class group of $A$ which is a group consisting of all the isomorphism classes of divisorial ideals of $A$. Recall that an $A$-module $M$ is called an $A$-lattice if there are free $A$-modules $F$ and $F^{\prime}$ with the same finite rank such that $F \subseteq M \subseteq F^{\prime}$. An $A$-lattice $M$ is called reflexive if the natural mapping $M \rightarrow M^{* *}$ is an isomorphism, where $(-)^{*}$ denotes $\operatorname{Hom}_{A}(-, A)$. A divisorial ideal is nothing but a reflexive $A$-lattice of rank one. Note that the multiplication in $C \ell(A)$ is defined by $[I] \cdot[J]=\left[\left(I \otimes_{A} J\right)^{* *}\right]$. See [3] or [6] for the details.

Proposition 4.1. Let $A$ be a Krull domain and we denote by $\operatorname{Ref}(A)$ the full subcategory of A-Mod consisting of all reflexive A-lattices. Then we have an equality

$$
\operatorname{Pic}(\operatorname{Ref}(A))=C \ell(A) \text {. }
$$

Proof. For any $[M] \in \operatorname{Pic}(\operatorname{Ref}(A))$, there exists an $\left[M^{\prime}\right] \in \operatorname{Pic}(\operatorname{Ref}(A))$ such that $\left.1_{\operatorname{Ref}(A)} \cong \operatorname{Hom}_{A}\left(M \otimes_{A} M^{\prime},-\right)\right|_{\operatorname{Ref}(A)}$ as functors on $\operatorname{Ref}(A)$. Since 
any $A$-homomorphism from $M \otimes_{A} M^{\prime}$ to a reflexive module factors through the natural $A$-homomorphism $M \otimes_{A} M^{\prime} \rightarrow\left(M \otimes_{A} M^{\prime}\right)^{* *}$, we have isomorphisms of functors

$$
\left.\left.1_{\operatorname{Ref}(A)} \cong \operatorname{Hom}_{A}\left(M \otimes_{A} M^{\prime},-\right)\right|_{\operatorname{Ref}(A)} \cong \operatorname{Hom}_{A}\left(\left(M \otimes_{A} M^{\prime}\right)^{* *},-\right)\right|_{\operatorname{Ref}(A)} .
$$

Since $\left(M \otimes_{A} M^{\prime}\right)^{* *}$ is again a reflexive $A$-lattice, we conclude that $\left(M \otimes_{A}\right.$ $\left.M^{\prime}\right)^{* *} \cong A$ by Yoneda's lemma. This implies that the reflexive $A$-lattice $M$ has rank one, thus $M$ is a divisorial ideal and $[M] \in C \ell(A)$.

On the other hand, we shall show that $\left.\operatorname{Hom}_{A}(I,-)\right|_{\operatorname{Ref}(A)}$ is a well-defined automorphism for any divisorial fractional ideal $I$ of $A$, hence that $[I] \in$ $\operatorname{Pic}(\operatorname{Ref}(A))$.

First of all we remark from Bourbaki [3, Chapter VII, §2] or Fossum [6, Chapter $1, \S 5]$ that an $A$-lattice $M$ is reflexive if and only if the equality $M=\bigcap_{\mathfrak{p} \in H(A)} M_{\mathfrak{p}}$ holds, where $H(A)$ is the set of all prime ideals of height one. Secondly we note that that the equality

$$
\operatorname{Hom}_{A}(X, Y)=\bigcap_{\mathfrak{p} \in H(A)} \operatorname{Hom}_{A}\left(X_{\mathfrak{p}}, Y_{\mathfrak{p}}\right)
$$

holds for $X, Y \in \operatorname{Ref}(A)$. In fact, any $f \in \bigcap_{\mathfrak{p} \in H(A)} \operatorname{Hom}_{A}\left(X_{\mathfrak{p}}, Y_{\mathfrak{p}}\right)$ maps $X$ to $Y_{\mathfrak{p}}$ for all $\mathfrak{p} \in H(A)$, hence $f(X) \subseteq \bigcap_{\mathfrak{p} \in H(R)} Y_{\mathfrak{p}}=Y$, and thus $f \in$ $\operatorname{Hom}_{A}(X, Y)$. Combining these two claims one can see that $\operatorname{Hom}_{A}(I, X)$ is a reflexive lattice for all $X \in \operatorname{Ref}(A)$. Hence $\operatorname{Hom}_{A}(I,-)$ yields a functor from $\operatorname{Ref}(A)$ to itself. Since $I$ is a divisorial ideal, there exists a divisorial ideal $J$ with $[J]=-[I]$ in $C \ell(A)$, i.e., $\left(I \otimes_{A} J\right)^{* *} \cong A$. Then there are isomorphisms of functors on $\operatorname{Ref}(A)$;

$$
\begin{aligned}
\left.\operatorname{Hom}_{A}\left(J, \operatorname{Hom}_{A}(I,-)\right)\right|_{\operatorname{Ref}(A)} & \left.\cong \operatorname{Hom}_{A}\left(I \otimes_{A} J,-\right)\right|_{\operatorname{Ref}(A)} \\
& \left.\cong \operatorname{Hom}_{A}\left(\left(I \otimes_{A} J\right)^{* *},-\right)\right|_{\operatorname{Ref}(A)} \\
& \cong 1_{\operatorname{Ref}(A)} .
\end{aligned}
$$

This shows that $\left.\operatorname{Hom}(I,-)\right|_{\operatorname{Ref}(A)}$ is an automorphism on $\operatorname{Ref}(A)$ as desired.

In a Noetherian case we have the following example.

Proposition 4.2. Let A be a Noetherian normal domain. We denote by $\operatorname{ref}(A)$ the full subcategory of A-Mod consisting of all finitely generated reflexive A-modules. Then we have the following equality.

$$
\operatorname{Pic}(\operatorname{ref}(A))=C \ell(A)
$$


Proof. In fact, if $A$ is a Noetherian Krull domain, then all reflexive $A$ lattices are finitely generated, hence we have $\operatorname{Ref}(A)=\operatorname{ref}(A)$.

Let $A$ be an integral domain. The torsion part $t(M)$ of an $A$-module $M$ is defined to be

$$
t(M)=\{m \in M \mid a m=0 \text { for some non-zero element } a \in A\} .
$$

An $A$-module $M$ is called torsion-free if $t(M)=0$. Note that $M / t(M)$ is always torsion-free for any $A$-module $M$.

Proposition 4.3. Let A be a commutative integral domain. We denote by $\operatorname{Tf}(A)$ (resp. $\operatorname{tf}(A))$ the full subcategory of A-Mod consisting of all torsionfree A-modules (resp. all finitely generated torsion-free A-modules). Then we have the equalities

$$
\operatorname{Pic}(\operatorname{Tf}(A))=\operatorname{Pic}(\operatorname{tf}(A))=\operatorname{Pic} A .
$$

Proof. Since $\operatorname{Tf}(A)$ (resp.tf $(A))$ is additively closed, the inclusion Pic $A \subseteq$ $\operatorname{Pic}(\operatorname{Tf}(A))$ (resp. Pic $A \subseteq \operatorname{Pic}(\operatorname{tf}(A)))$ holds by Lemma 3.5.

To prove the other inclusion, assume $[M] \in \operatorname{Pic}(\operatorname{Tf}(A))$ (resp. $[M] \in$ $\operatorname{Pic}(\operatorname{tf}(A)))$. We have only to show that $M$ is an invertible $A$-module. Take a torsion-free $A$-module $\left[M^{\prime}\right] \in \operatorname{Pic}(\operatorname{Tf}(A))\left(\operatorname{resp} .\left[M^{\prime}\right] \in \operatorname{Pic}(\operatorname{tf}(A))\right)$ such that $\operatorname{Hom}_{A}\left(M \otimes_{A} M^{\prime},-\right) \cong 1$ as functors on $\operatorname{Tf}(A)$ (resp. $\left.\operatorname{tf}(A)\right)$. We define the torsion-free tensor product by

$$
M \bar{\otimes}_{A} M^{\prime}:=\left(M \otimes_{A} M^{\prime}\right) / t\left(M \otimes_{A} M^{\prime}\right) .
$$

It is easy to see that every $A$-homomorphism from $M \otimes_{A} M^{\prime}$ to a torsion-free $A$-module factors through the natural surjection $M \otimes_{A} M^{\prime} \rightarrow M \bar{\otimes}_{A} M^{\prime}$. Hence the above isomorphism of functors induces an isomorphism

$$
\operatorname{Hom}_{A}\left(M \bar{\otimes}_{A} M^{\prime},-\right) \cong 1,
$$

as functors on $\operatorname{Tf}(A)$ (resp. $\operatorname{tf}(A)$ ). Since $M \bar{\otimes}_{A} M^{\prime}$ is torsion-free, it follows from Yoneda's lemma that $M \bar{\otimes}_{A} M^{\prime} \cong A$ as $A$-modules. Note from this isomorphism that $M$ has rank one.

Now we prove the following claim.

Claim. Assume that $M \bar{\otimes}_{A} M^{\prime} \cong A$ for torsion-free A-modules $M$ and $M^{\prime}$. Then we have $M_{\mathfrak{p}} \cong M_{\mathfrak{p}}^{\prime} \cong A_{\mathfrak{p}}$ for any prime ideal $\mathfrak{p}$ of $A$.

In fact, take an element $\sum_{i=1}^{r} m_{i} \otimes m_{i}^{\prime} \in M \otimes_{A} M^{\prime}$ which maps to 1 by the natural epimorphism

$$
\pi: M \otimes_{A} M^{\prime} \rightarrow M \bar{\otimes}_{A} M^{\prime} \cong A .
$$


Localizing at $\mathfrak{p}$, we have the $A_{\mathfrak{p}}$-homomorphism

$$
\pi_{\mathfrak{p}}: M_{\mathfrak{p}} \otimes_{A_{\mathfrak{p}}} M_{\mathfrak{p}}^{\prime} \cong\left(M \otimes_{A} M^{\prime}\right)_{\mathfrak{p}} \rightarrow\left(M \bar{\otimes}_{A} M^{\prime}\right)_{\mathfrak{p}} \cong A_{\mathfrak{p}}
$$

Since $A_{\mathfrak{p}}$ is a local ring, we may assume that $m_{1} / 1 \otimes m_{1}^{\prime} / 1 \in M_{\mathfrak{p}} \otimes_{A_{\mathfrak{p}}} M_{\mathfrak{p}}^{\prime}$ is mapped to a unit element of $A_{\mathfrak{p}}$ by $\pi_{\mathfrak{p}}$. Let $f_{\mathfrak{p}}$ be an $A_{\mathfrak{p}}$-homomorphism from $A_{\mathfrak{p}}$ to $M_{\mathfrak{p}}^{\prime}$ defined by $f(a)=a \cdot\left(m_{1}^{\prime} / 1\right)\left(a \in A_{\mathfrak{p}}\right)$. Taking the tensor product of $M_{\mathfrak{p}}$ with $f_{\mathfrak{p}}$ and making the composition with $\pi_{\mathfrak{p}}$, we obtain an $A_{\mathfrak{p}}$-homomorphism

$$
M_{\mathfrak{p}}=M_{\mathfrak{p}} \otimes_{A_{\mathfrak{p}}} A_{\mathfrak{p}} \stackrel{M_{\mathfrak{p}} \otimes f_{\mathfrak{p}}}{\longrightarrow} M_{\mathfrak{p}} \otimes_{A_{\mathfrak{p}}} M_{\mathfrak{p}}^{\prime} \stackrel{\pi_{\mathfrak{p}}}{\longrightarrow} A_{\mathfrak{p}},
$$

which we denote by $g_{\mathfrak{p}}$. Note that $g_{\mathfrak{p}}$ is an epimorphism, since $g_{\mathfrak{p}}\left(m_{1} / 1\right)=$ $\pi_{\mathfrak{p}}\left(m_{1} / 1 \otimes m_{1}^{\prime} / 1\right)$ is a unit of $A_{\mathfrak{p}}$. Therefore we see that $M_{\mathfrak{p}} \cong A_{\mathfrak{p}} \oplus K$ as $A_{\mathfrak{p}}$-modules, where $K=\operatorname{ker}\left(g_{\mathfrak{p}}\right)$. Since $M_{\mathfrak{p}}$ has rank one, $K$ must be a torsion $A_{\mathfrak{p}}$-module. However $M_{\mathfrak{p}}$ is a torsion-free $A_{\mathfrak{p}}$-module, hence we have $K=0$. Thus $M_{\mathfrak{p}} \cong A_{\mathfrak{p}}$. This completes the proof of the claim.

Now from the claim we have that

$$
\left(M \otimes_{A} M^{\prime}\right)_{\mathfrak{p}} \cong M_{\mathfrak{p}} \otimes_{A_{\mathfrak{p}}} M_{\mathfrak{p}}^{\prime} \cong A_{\mathfrak{p}}
$$

which is a torsion-free $A_{\mathfrak{p}}$-module. It follows from this that $t\left(M \otimes_{A} M^{\prime}\right)_{\mathfrak{p}}=0$. Since this equality holds for any prime ideal $\mathfrak{p}$, we have that $t\left(M \otimes_{A} M^{\prime}\right)=0$. Therefore, we have $M \otimes_{A} M^{\prime} \cong M \bar{\otimes}_{A} M^{\prime} \cong A$, which shows that $M$ is an invertible $A$-module.

Let $(A, \mathfrak{m})$ be a Noetherian local ring. We consider the full subcategory $d^{\geq 1}(A)$ of $A$-Mod which consists of all the finitely generated $A$-modules $M$ with depth $M \geq 1$. Denote by $H_{\mathfrak{m}}^{0}(M)$ the 0 th local cohomology module of an $A$-module $M$, i.e.,

$$
H_{\mathfrak{m}}^{0}(M)=\left\{x \in M \mid \mathfrak{m}^{n} x=0 \text { for some natural number } n\right\} .
$$

Note that a finitely generated $A$-module $M$ is an object of $d^{\geq 1}(A)$ if and only if $H_{\mathfrak{m}}^{0}(M)=0$. Moreover, for any finitely generated $A$-module $M, M / H_{\mathfrak{m}}^{0}(M)$ is always an object of $d^{\geq 1}(A)$.

Proposition 4.4. Let $A$ be a Noetherian local ring with depth $A>0$. Then $\operatorname{Pic}\left(d^{\geq 1}(A)\right)$ is a trivial group.

Proof. Let $[M] \in \operatorname{Pic}\left(d^{\geq 1}(A)\right)$, and we shall show that $M \cong A$ as $A$ modules. Take an element $\left[M^{\prime}\right] \in \operatorname{Pic}\left(d^{\geq 1}(A)\right)$ such that

$$
\left.1_{d \geq 1}(A) \cong \operatorname{Hom}_{A}\left(M \otimes_{A} M^{\prime},-\right)\right|_{d \geq 1}(A) .
$$


We define $M \tilde{\otimes}_{A} M^{\prime}$ as the residue module $M \otimes_{A} M^{\prime} / H_{\mathfrak{m}}^{0}\left(M \otimes_{A} M^{\prime}\right)$. Then $M \tilde{\otimes}_{A} M^{\prime} \in d^{\geq 1}(A)$ and it is easy to see that every $A$-homomorphism from $M \otimes_{A} M^{\prime}$ to an object of $d^{\geq 1}(A)$ factors through the natural surjection $M \otimes_{A}$ $M^{\prime} \rightarrow M \tilde{\otimes}_{A} M^{\prime}$. Hence the above isomorphism of functors induces an isomorphism

$$
\left.1_{d \geq 1}(A) \cong \operatorname{Hom}_{A}\left(M \tilde{\otimes}_{A} M^{\prime},-\right)\right|_{d \geq 1}(A) .
$$

Since $M \tilde{\otimes}_{A} M^{\prime} \in d^{\geq 1}(A)$, it follows from Yoneda's lemma that there is an isomorphism $M \tilde{\otimes}_{A} M^{\prime} \cong A$ as $A$-modules. Thus $M \otimes_{A} M^{\prime}$ is isomorphic to $A \oplus H_{\mathfrak{m}}^{0}\left(M \otimes_{A} M^{\prime}\right)$. We denote by $\pi$ the natural projection from $M \otimes_{A} M^{\prime}$ to $A$. Note that $\operatorname{ker}(\pi) \cong H_{\mathfrak{m}}^{0}\left(M \otimes_{A} M^{\prime}\right)$ is of finite length, hence the localized mapping $\pi_{\mathfrak{p}}: M_{\mathfrak{p}} \otimes_{A_{\mathfrak{p}}} M_{\mathfrak{p}}^{\prime} \cong\left(M \otimes_{A} M^{\prime}\right)_{\mathfrak{p}} \rightarrow A_{\mathfrak{p}}$ is an isomorphism of $A_{\mathfrak{p}}-$ modules for any prime ideal $\mathfrak{p} \neq \mathfrak{m}$ of $A$. Thus $M_{\mathfrak{p}}$ is an invertible $A_{\mathfrak{p}}$-module. Since $A_{\mathfrak{p}}$ is a local ring, it forces that $M_{\mathfrak{p}} \cong A_{\mathfrak{p}}$ as $A_{\mathfrak{p}}$-modules.

Let $\sum_{i=1}^{n} m_{i} \otimes m_{i}^{\prime}$ be an element of $M \otimes_{A} M^{\prime}$ such that $\pi\left(\sum_{i=1}^{n} m_{i} \otimes m_{i}^{\prime}\right)=$ 1. Since $A$ is a local ring, we may assume that $\pi\left(m_{1} \otimes m_{1}^{\prime}\right)$ is a unit element in $A$. We define an $A$-homomorphism $f: A \rightarrow M^{\prime}$ by $f(a)=a m_{1}^{\prime}(a \in A)$. Taking the tensor product with $M$ and making the composition with $\pi$, we have an $A$-homomorphism

$$
M \stackrel{M \otimes f}{\longrightarrow} M \otimes_{A} M^{\prime} \stackrel{\pi}{\longrightarrow} A,
$$

which we denote by $g$. Then $g$ is an epimorphism, since $g\left(m_{1}\right)=\pi\left(m_{1} \otimes m_{1}^{\prime}\right)$ is a unit of $A$. For any prime ideal $\mathfrak{p}$ with $\mathfrak{p} \neq \mathfrak{m}$, since the localized mapping $g_{\mathfrak{p}}: M_{\mathfrak{p}} \rightarrow A_{\mathfrak{p}}$ is surjective and since $M_{\mathfrak{p}} \cong A_{\mathfrak{p}}$, we can see that $g_{\mathfrak{p}}$ is an isomorphism of $A_{\mathfrak{p}}$-modules. This implies that $\operatorname{ker}(g)$ is an $A$-module of finite length. Since depth $M \geq 1$, the submodule $\operatorname{ker}(g)$ of $M$ of finite length must be 0 . Therefore $g: M \rightarrow A$ is an isomorphism of $A$-modules.

Now let $(A, \mathfrak{m})$ be a Noetherian complete local ring, and we denote by $E$ the injective envelope of the $A$-module $A / \mathrm{m}$. An $A$-module $M$ is called Matlisreflexive if the natural mapping $M \rightarrow M^{\vee \vee}$ is an isomorphism, where (-) $)^{\vee}$ denotes $\operatorname{Hom}_{A}(-, E)$. It is well known by [7] that every Noetherian or Artinian $A$-module is Matlis-reflexive. We denote by $\operatorname{Mat}(A)$ the full subcategory of $A$-Mod consisting of all the Matlis-reflexive $A$-modules. Note from [5] that an $A$-module $M$ belongs to $\operatorname{Mat}(A)$ if and only if there is a short exact sequence

$$
0 \longrightarrow N \longrightarrow M \longrightarrow L \longrightarrow 0,
$$

where $N$ is a Noetherian $A$-module and $L$ is an Artinian $A$-module. It is easy to see that any submodule and any quotient module of a Matlis-reflexive module is again Matlis-reflexive. Hence the subcategory $\operatorname{Mat}(A)$ is an abelian category. It is also easy to see that $\operatorname{Mat}(A)$ is additively closed. 
Proposition 4.5. Let $(A, \mathfrak{m})$ be a Noetherian complete local ring. Then $\operatorname{Pic}(\operatorname{Mat}(A))$ is a trivial group.

Proof. Let $[M] \in \operatorname{Pic}(\operatorname{Mat}(A))$ be an arbitrary element. It is enough to show that $M$ is an invertible $A$-module. Take an inverse element $[N]=[M]^{-1}$ in $\operatorname{Pic}(\operatorname{Mat}(A))$ with $N \in \operatorname{Mat}(A)$. Setting $L=M \otimes_{A} N$, from the definition we have an isomorphism of functors

$$
\left.\operatorname{Hom}_{A}(L,-)\right|_{\operatorname{Mat}(A)} \cong 1_{\operatorname{Mat}(A)} .
$$

We have only to show that $L \cong A$ from this isomorphism. Since $E \in \operatorname{Mat}(A)$, we have an isomorphism $L^{\vee}=\operatorname{Hom}_{A}(L, E) \cong E$. Since the natural mapping $L \rightarrow L^{\vee \vee}$ is always an injection, we have an embedding $L \rightarrow L^{\vee \vee} \cong E^{\vee} \cong$ $A$. Therefore $L$ is isomorphic to an ideal of $A$, hence it is finitely generated. On the other hand, we have isomorphisms of $A$-modules:

$$
\begin{aligned}
\operatorname{Hom}_{A}\left(L \otimes_{A} A / \mathfrak{m}, E\right) & \cong \operatorname{Hom}_{A}\left(A / \mathfrak{m}, L^{\vee}\right) \\
& \cong \operatorname{Hom}_{A}(A / \mathfrak{m}, E) \cong A / \mathfrak{m} .
\end{aligned}
$$

Since $L \otimes_{A} A / \mathrm{m}$ is finitely generated, we have

$$
L \otimes_{A} A / \mathfrak{m} \cong\left(L \otimes_{A} A / \mathfrak{m}\right)^{\vee \vee} \cong(A / \mathfrak{m})^{\vee} \cong A / \mathfrak{m} .
$$

This shows that $L$ is generated by a single element by Nakayama's lemma. Hence we may write $L \cong A / I$ for some ideal $I$ of $A$. Since $A \in \operatorname{Mat}(A)$, we also have an isomorphism of $A$-modules:

$$
\operatorname{Hom}_{A}(L, A) \cong A .
$$

Since $I$ annihilates the left hand side, we have $I A=0$, hence $L \cong A$ as desired.

Remark 4.6. Any subgroups of Pic $A$ (resp. $C \ell(A)$ ) will appear as the Picard groups of some additive full subcategories. In fact, it is not difficult to see that the following claims hold. (We leave their proofs to the reader.)

(1) Let $A$ be a commutative ring. For any additive full subcategory $\mathbb{5}$ of $\operatorname{proj}(A)$ with $A \in \mathfrak{C}, \operatorname{Pic}(\mathfrak{S})$ is naturally a subgroup of Pic $A$. Conversely, for any subgroup $G$ of Pic $A$, there exists an additive full subcategory $\mathbb{S}$ of $\operatorname{proj}(A)$ such that $\operatorname{Pic}(\mathfrak{C})=G$.

(2) Let $A$ be a Krull domain. For any additive full subcategory $\mathfrak{C}$ of $\operatorname{Ref}(A)$ such that $A \in \mathfrak{C}, \operatorname{Pic}(\mathfrak{S})$ is naturally a subgroup of $C \ell(A)$. Conversely, for any subgroup $G$ of $C \ell(A)$, there exists an additive full subcategory $\mathfrak{S}$ of $\operatorname{Ref}(A)$ such that $\operatorname{Pic}(\mathfrak{C})=G$. 


\section{Structure of the group of $\boldsymbol{k}$-linear automorphisms}

Let $k$ be a commutative ring and let $A$ be a commutative $k$-algebra. We denote by $\operatorname{Aut}_{k \text {-alg }}(A)$ the group of all the $k$-algebra automorphisms of $A$. For an automorphism $\sigma \in \operatorname{Aut}_{k \text {-alg }}(A)$, we can define a covariant $k$-linear functor $\sigma_{*}: A$-Mod $\rightarrow A$-Mod as in the following manner. For each $A$-module $M$, we define $\sigma_{*}(M)$ to be $\sigma_{*} M=M$ as an abelian group on which the $A$-module structure is given by

$$
a \circ m=\sigma^{-1}(a) m \quad(a \in A, m \in M) .
$$

For an $A$-homomorphism $f: M \rightarrow N$ of $A$-modules, we define $\sigma_{*} f: \sigma_{*} M \rightarrow$ $\sigma_{*} N$ to be the same mapping as $f$. Note that $\sigma_{*} f$ is an $A$-homomorphism, since the equalities $\left(\sigma_{*} f\right)(a \circ m)=f\left(\sigma^{-1}(a) m\right)=\sigma^{-1}(a) f(m)=a \circ \sigma_{*} f(m)$ hold for $a \in A$ and $m \in M$. It is easily verified that the equality $(\sigma \tau)_{*}=\sigma_{*} \tau_{*}$ holds for $\sigma, \tau \in \operatorname{Aut}_{k \text {-alg }}(A)$. In fact, we see that $a \circ m=(\sigma \tau)^{-1}(a) m$ on $(\sigma \tau)_{*} M$, on the other hand $a \circ m=\tau^{-1}\left(\sigma^{-1}(a)\right) m$ on $\sigma_{*} \tau_{*} M$. Since $\sigma_{*}\left(\sigma^{-1}\right)_{*}$ is the identity functor on $A$-Mod, the $k$-linear functor $\sigma_{*}: A$-Mod $\rightarrow A$-Mod is an equivalence of the category.

Let $\subseteq$ be any subcategory of $A$-Mod. We denote by Aut ${ }_{k}(\mathfrak{S})$ the group of all the isomorphism classes of $k$-linear automorphism of $\mathfrak{E}$, i.e., an element of $\operatorname{Aut}_{k}(\mathfrak{S})$ is the isomorphism class $[F]$ of a $k$-linear equivalence $F: \mathfrak{S} \rightarrow \mathfrak{S}$, and the multiplication in $\mathrm{Aut}_{k}(\mathfrak{S})$ is defined to be the composition of functors.

Under these notations we have the following lemma.

Lemma 5.1. There is an injective group homomorphism $\Psi: \operatorname{Aut}_{k \text {-alg }}(A) \rightarrow$ $\operatorname{Aut}_{k}\left(A\right.$-Mod) which maps $\sigma$ to the isomorphism class of $\sigma_{*}$.

Proof. It is easy to see from the observation above that $\Psi$ is a group homomorphism. we have only to show that it is injective. For this, it is enough to prove the following claim.

CLAIM. If $\sigma_{*}$ is isomorphic to the identity functor on A-Mod, then the $k$-algebra automorphism $\sigma$ is the identity mapping.

In fact, letting $\varphi: 1_{A \text {-Mod }} \rightarrow \sigma_{*}$ be an isomorphism of functors, for any $a \in A$ we have the following commutative diagram, from the definition of $\sigma_{*}$.

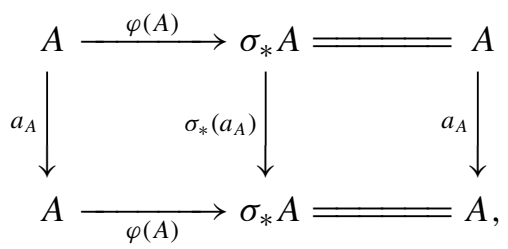

where $a_{A}$ denotes the multiplication mapping by $a$ on $A$. Thus it follows the equality $\varphi(A)(a)=a \cdot \varphi(A)(1)$ as an element of $\sigma_{*} A=A$. On the other hand, 
since $\varphi(A)$ is an $A$-homomorphism, we must have $\varphi(A)(a)=a \circ \varphi(A)(1)=$ $\sigma^{-1}(a) \cdot \varphi(A)(1)$. Therefore we have $a=\sigma^{-1}(a)$. Since this holds for all $a \in A$, we conclude that $\sigma$ is the identity mapping.

It is worth noting that $\Psi$ may not be surjective.

Definition 5.2. Let $C$ be an additive full subcategory of $A$-Mod and let $\sigma \in \operatorname{Aut}_{k \text {-alg }}(A)$. Then $\mathbb{S}$ is called $\sigma$-stable if $\sigma_{*}$ gives an equivalence $\mathfrak{S} \rightarrow \mathfrak{S}$, i.e., $\sigma_{*}(M) \in \mathbb{C}$ and $\left(\sigma^{-1}\right)_{*}(M) \in \mathbb{C}$ whenever $M \in \mathbb{C}$. We also say that $\mathbb{C}$ is stable under $\operatorname{Aut}_{k \text {-alg }}(A)$ if $\mathbb{C}$ is $\sigma$-stable for all $\sigma \in \operatorname{Aut}_{k \text {-alg }}(A)$.

Example 5.3. The following subcategories of $A$-Mod are stable under $\operatorname{Aut}_{k \text {-alg }}(A)$.

(1) $A$-mod, $\operatorname{proj}(A)$ and $\operatorname{Proj}(A) .($ See Example 3.8.)

(2) $\operatorname{Ref}(A)$ in case $A$ is a Krull domain, and $\operatorname{ref}(A)$ in case $A$ is a Noetherian normal domain. (See Propositions 4.1 and 4.2.)

(3) $\operatorname{Tf}(A)$ and $\operatorname{tf}(A)$ in case $A$ is an integral domain. (See Proposition 4.3.)

(4) the full subcategory $d^{\geq i}(A)$ which consists of all the finitely generated $A$-modules $M$ with depth $M \geq i$ when $A$ is a Noetherian local ring with depth $A \geq i$, where $i$ is any natural number. (Cf. Proposition 4.4.)

(5) $\operatorname{Mat}(A)$ in case $A$ is a Noetherian complete local ring. (See Proposition 4.5.)

(6) $\mathrm{CM}(A)$ in case $A$ is a Cohen-Macaulay local ring. (See $\$ 5$ below.)

Notice that an additive full subcategory of $A$-Mod is not necessarily stable under $\operatorname{Aut}_{k \text {-alg }}(A)$.

Regarding $A$ as an $A$-algebra, we can consider the group $\mathrm{Aut}_{A}(\mathfrak{C})$ for an additive full subcategory 5 of $A$-Mod. Since any $A$-linear functor is a $k$ linear functor in an obvious way, there is a group homomorphism Aut $_{A}$ (5) $\rightarrow$ $\operatorname{Aut}_{k}(\mathbb{5})$ whose kernel consists of a single element that is the class of the identity functor on $\sqrt{5}$. In such a way, $\operatorname{Aut}_{A}(\mathfrak{C})$ is naturally a subgroup of $\operatorname{Aut}_{k}(A)$.

If the additive full subcategory $\sqrt{5}$ contains $A$ as an object, then we have shown in Lemma 3.3 that $\operatorname{Pic}(\mathfrak{C})$ is isomorphic to $\operatorname{Aut}_{A}(\mathfrak{C})$. Thus we obtain the following lemma.

LEMMA 5.4. Let $A$ be a commutative algebra over a commutative ring $k$, and let $\mathbb{5}$ be an additive full subcategory of $A$-Mod with $A \in \mathbb{E}$. Then Pic(5) is naturally a subgroup of $\mathrm{Aut}_{k}(\mathfrak{S})$ by mapping $[M] \in \mathrm{Pic}(\mathfrak{5})$ to the class of automorphism $\left.\operatorname{Hom}_{A}(M,-)\right|_{\mathfrak{c}}$.

We should remark that Pic(C) is an abelian group while $\mathrm{Aut}_{k}(\mathfrak{C})$ is not. 
DEFINITION 5.5. In the rest of the paper, we always assume that the additive full subcategory 5 is stable under $\operatorname{Aut}_{k \text {-alg }}(A)$. Then by definition there is a group homomorphism

$$
\left.\Psi\right|_{\mathfrak{S}}: \operatorname{Aut}_{k \text {-alg }}(A) \rightarrow \operatorname{Aut}_{k}(\mathfrak{C})
$$

which maps $\sigma$ to the class of $\left.\sigma_{*}\right|_{\mathfrak{c}}$. We denote the image of $\left.\Psi\right|_{\mathfrak{S}}$ by $\mathrm{I}_{k}(\mathfrak{C})$. Note that $\mathrm{I}_{k}(\mathfrak{C})$ is a subgroup of $\mathrm{Aut}_{k}(\mathfrak{S})$. We call $\mathrm{I}_{k}(\mathfrak{S})$ the subgroup of $k$-linear automorphisms of $\mathfrak{E}$ induced by $k$-algebra automorphisms.

We should note the following lemma.

LEMMA 5.6. Let 5 be an additive full subcategory of A-Mod which is stable under $\operatorname{Aut}_{k \text {-alg }}(A)$ and $A \in \mathfrak{C}$. Then the group homomorphism $\left.\Psi\right|_{\mathfrak{E}}$ : $\operatorname{Aut}_{k-\mathrm{alg}}(A) \rightarrow \operatorname{Aut}_{k}(\mathfrak{S})$ is injective. In particular, $\operatorname{Aut}_{k \text {-alg }}(A) \cong \mathrm{I}_{k}(\mathfrak{S})$ as groups.

Proof. To show the injectivity of $\left.\Psi\right|_{\mathfrak{S}}$, it is enough to prove that $\sigma_{*} \cong 1_{\mathfrak{S}}$ as functors on 5 only if $\sigma$ is the identity mapping on $A$. But the proof of this will be the same as that of the claim in the proof of Lemma 5.1.

In this way we obtain the subgroups $\mathrm{Aut}_{A}(\mathfrak{S})$ and $\mathrm{I}_{k}(\mathfrak{S})$ in $\mathrm{Aut}_{k}(\mathfrak{5})$ which are isomorphic as groups to $\mathrm{Pic}(5)$ and $\operatorname{Aut}_{k \text {-alg }}(A)$ respectively. For $[M] \in \operatorname{Pic}(\mathbb{C})$ and $\sigma \in \operatorname{Aut}_{k \text {-alg }}(A)$, it is easy to see that $\sigma_{*} M$ defines an element of Pic(C), hence the group $\operatorname{Aut}_{k \text {-alg }}(A)$ acts on $\mathrm{Pic}(\mathfrak{S})$. In fact we can show the following lemma.

Lemma 5.7. Let $[M] \in \operatorname{Pic}(\mathcal{C})$ and let $\sigma \in \operatorname{Aut}_{k \text {-alg }}(A)$ as above. Then there is an isomorphism of functors on 5

$$
\left.\left.\left.\left.\operatorname{Hom}_{A}\left(\sigma_{*} M,-\right)\right|_{\Subset} \cong \sigma_{*}\right|_{\subsetneq} \circ \operatorname{Hom}_{A}(M,-)\right|_{\Subset} \circ\left(\sigma^{-1}\right)_{*}\right|_{\Subset}
$$

Proof. It is enough to show that there is a natural isomorphism

$$
\operatorname{Hom}_{A}\left(\sigma_{*} M, X\right) \cong \sigma_{*} \operatorname{Hom}_{A}\left(M,\left(\sigma^{-1}\right)_{*} X\right)
$$

as $A$-modules for any $X \in \mathbb{C}$. But the both sides are the set of homomorphisms of abelian groups $f: M \rightarrow X$ satisfying $f\left(\sigma^{-1}(a) m\right)=a f(m)$ for all $a \in A$ and $m \in M$, on which the $A$-module structure is given as $(a \circ f)(m)=$ $f\left(\sigma^{-1}(a) m\right)$ for $a \in A$ and $m \in M$.

This lemma shows that under the isomorphism Pic(5) $\cong \operatorname{Aut}_{A}(\mathfrak{5})$ in Lemma 3.3, an element $\left[\sigma_{*} M\right] \in \operatorname{Pic}(\mathfrak{C})$ corresponds to the product of functors $\left.\left.\left.\sigma_{*}\right|_{\varsigma} \circ \operatorname{Hom}_{A}(M,-)\right|_{\text {๘ }} \circ\left(\sigma^{-1}\right)_{*}\right|_{\text {ธ }}$. 
Lemma 5.8. Let $[F] \in$ Aut $_{k}($ (5) be an arbitrary element. Then $F$ is isomorphic to the product of functors

$$
\left.\left.\sigma_{*}\right|_{\mathfrak{c}} \circ \operatorname{Hom}_{A}(M,-)\right|_{\mathfrak{s}},
$$

for some $\sigma \in \mathrm{Aut}_{k \text {-alg }}(\mathfrak{5})$ and $[M] \in \mathrm{Pic}(\mathfrak{5})$. In particular, we have the equality $\operatorname{Aut}_{k}(A)=\mathrm{I}_{k}(\mathfrak{C}) \cdot \operatorname{Aut}_{A}($ (C) as a group.

Proof. By Theorem 2.5, given an equivalence $F$, we have a $k$-algebra automorphism $\tau$ of $A$ and an $A$-module $M \in \mathfrak{S}$ such that there is an isomorphism of functors $\left.F \cong \operatorname{Hom}_{A}\left(M_{\tau},-\right)\right|_{\mathfrak{c}}$. It is easily observed from the definition of $M_{\tau}$ (Definition 2.4) that the right hand side equals the product of functors $\left.\left.\left(\tau^{-1}\right)_{*}\right|_{\varsigma} \circ \operatorname{Hom}_{A}(M,-)\right|_{\varsigma}$. Since $F$ and $\left.\left(\tau^{-1}\right)_{*}\right|_{\varsigma}$ are equivalences, so is $\left.\operatorname{Hom}_{A}(M,-)\right|_{\mathfrak{c}}$, hence $[M] \in \operatorname{Pic}(\mathfrak{c})$.

Now we are able to prove the main theorem in this paper.

THEOREM 5.9. Let $k$ be a commutative ring and let $A$ be a commutative $k$ algebra. Assume that an additive full subcategory 5 of A-Mod is stable under $\operatorname{Aut}_{k \text {-alg }}(A)$ and $A \in \mathcal{C}$. Then there is an isomorphism of groups

$$
\operatorname{Aut}_{k}(\mathfrak{C}) \cong \operatorname{Aut}_{k-\operatorname{alg}}(A) \ltimes \operatorname{Pic}(\mathfrak{C}) .
$$

Proof. Recall that the group $\operatorname{Aut}_{k \text {-alg }}(A)$ acts on the group Pic(C), i.e., an element $\sigma \in \operatorname{Aut}_{k \text {-alg }}(A)$ sends $[M] \in \operatorname{Pic}(5)$ to $\left[\sigma_{*} M\right]$. Therefore the semi-direct product $\operatorname{Aut}_{k \text {-alg }}(A) \ltimes \operatorname{Pic}(\mathfrak{S})$ is the set of pairs $(\sigma,[M])$ where $\sigma \in \operatorname{Aut}_{k \text {-alg }}(A)$ and $[M] \in \operatorname{Pic}(5)$, on which the multiplication is defined as

$$
(\sigma,[M]) \cdot(\tau,[N])=\left(\sigma \cdot \tau,\left[\left(\tau^{-1}\right)_{*} M\right] \cdot[N]\right) .
$$

Now we define a mapping

$$
\Phi: \operatorname{Aut}_{k \text {-alg }}(A) \ltimes \operatorname{Pic}(5) \rightarrow \operatorname{Aut}_{k}(\text { (5) }
$$

by sending $(\sigma,[M])$ to the class of the product of functors $\left.\left.\sigma_{*}\right|_{\mathfrak{c}} \circ \operatorname{Hom}_{A}(M,-)\right|_{\text {c }}$. We can verify that $\Phi$ is a homomorphism of groups by the following computations using Lemma 5.7.

$$
\begin{aligned}
\Phi(\sigma, & {[M]) \circ \Phi(\tau,[N]) } \\
& =\left.\left.\left.\left.\sigma_{*}\right|_{\mathfrak{S}} \circ \operatorname{Hom}_{A}(M,-)\right|_{\mathfrak{S}} \circ \tau_{*}\right|_{\mathfrak{S}} \circ \operatorname{Hom}_{A}(N,-)\right|_{\mathfrak{S}} \\
& =\left.\left.\left.\left.\sigma_{*}\right|_{\mathfrak{S}} \circ \tau_{*}\right|_{\mathfrak{S}} \circ \operatorname{Hom}_{A}\left(\left(\tau^{-1}\right)_{*} M,-\right)\right|_{\mathfrak{S}} \circ \operatorname{Hom}_{A}(N,-)\right|_{\mathfrak{S}} \\
& =\Phi\left(\sigma \cdot \tau,\left[\left(\tau^{-1}\right)_{*} M\right] \cdot[N]\right)
\end{aligned}
$$


It follows from Lemma 5.8 that $\Phi$ is a surjective mapping. It remains to show that $\Phi$ is injective. To prove this, assume that $\Phi(\sigma,[M])=\left.\sigma_{*}\right|_{\mathfrak{c}} \circ$ $\left.\operatorname{Hom}_{A}(M,-)\right|_{\mathfrak{c}}$ is isomorphic to the identity functor $1_{\mathfrak{c}}$. Then we have an isomorphism $\left.\left.\left(\sigma^{-1}\right)_{*}\right|_{\varsigma} \cong \operatorname{Hom}_{A}(M,-)\right|_{\mathfrak{c}}$, in particular, $\left.\left(\sigma^{-1}\right)_{*}\right|_{\varsigma}$ is an $A$ linear functor. Thus the following claim is sufficient to finish the proof.

Claim. Assume that $\left.\tau_{*}\right|_{\mathfrak{S}}$ is an A-linear functor on $\complement_{5}$ for $\tau \in \operatorname{Aut}_{k \text {-alg }}(A)$. Then $\tau$ is the identity mapping on A.

For any $a \in A$, the multiplication map $a_{A}$ on $A$ by $a$ is an $A$-homomorphism. Note that we can apply the functor $\left.\tau_{*}\right|_{\text {c }}$ to $a_{A}: A \rightarrow A$, because $A \in \mathcal{C}$. Since $\left.\tau_{*}\right|_{\mathrm{s}}$ is an $A$-linear functor, we have the equality

$$
\tau_{*}\left(a_{A}\right)=a_{\tau_{*} A} \cdot \tau_{*}\left(1_{A}\right)=a_{\tau_{*} A}
$$

as a mapping $\tau_{*} A \rightarrow \tau_{*} A$. Recall that $A=\tau_{*} A$ as abelian groups, and that $\tau_{*}\left(a_{A}\right)$ is the same as $a_{A}$ as a mapping on $A$. Evaluating the both ends of the equality above at $1 \in A=\tau_{*} A$, we see that $a=\tau_{*}\left(a_{A}\right)(1)=a_{\tau_{*} A}(1)=$ $\tau^{-1}(a)$. Thus the equality $\tau(a)=a$ holds for all $a \in A$. This completes the proof of the claim.

Corollary 5.10. Under the circumstances of Theorem 5.9, Pic(5) is naturally a normal subgroup of $\mathrm{Aut}_{k}(\mathrm{~S})$ and the residue class group $\operatorname{Aut}_{k}\left(\right.$ (5) / Pic(C) is isomorphic to $\operatorname{Aut}_{k \text {-alg }}(A)$.

\section{Automorphisms of the category of Cohen-Macaulay modules}

In this section, let $(A, \mathrm{~m})$ be a Cohen-Macaulay local $k$-algebra, i.e., $A$ is a Noetherian local $k$-algebra with maximal ideal $\mathfrak{m}$ and satisfies the equality depth $A=\operatorname{dim} A$. We are interested in the additive full subcategory $\operatorname{CM}(A)$ of $A$-mod consisting of all the maximal Cohen-Macaulay modules over $A$. Note that a finitely generated $A$-module $M$ belongs to $\mathrm{CM}(A)$ if and only if depth $M=\operatorname{dim} A$. (Cf. See [12].)

First we should note the following proposition as a direct consequence of Corollary 2.3, which says that the category $\mathrm{CM}(A)$ well reflects the $k$-algebra structure of $A$.

Proposition 6.1. Let $A$ and $B$ be Cohen-Macaulay local $k$-algebras. If $\mathrm{CM}(A)$ and $\mathrm{CM}(B)$ are isomorphic to each other as $k$-categories, then $A$ and $B$ are isomorphic to each other as $k$-algebras.

Now we shall prove Theorem 1.1 in the introduction.

Recall that $A$ is said to have only an isolated singularity if $A_{\mathfrak{p}}$ is a regular local ring for each prime ideal $\mathfrak{p}$ except $\mathfrak{m}$. See [12, Chapter 3 ]. Now, to 
prove Theorem 1.1, suppose that $A$ is a Cohen-Macaulay local ring having only an isolated singularity. If $\operatorname{dim} A=0$, then $\operatorname{CM}(A)=A$-mod. Hence it follows from Example 3.8 that $\operatorname{Pic}(\operatorname{CM}(A))=\operatorname{Pic} A$ which is a trivial group, since $A$ is a local ring. If $\operatorname{dim} A=1$, then $\operatorname{CM}(A)=d^{\geq 1}(A)$ and we have shown in Proposition 4.4 that $\operatorname{Pic}(\operatorname{CM}(A))$ is again a trivial group. On the other hand, if $\operatorname{dim} A=2$, then $A$ is a Noetherian normal domain and $\mathrm{CM}(A)=\operatorname{ref}(A)$. Therefore we have the equality $\operatorname{Pic}(\mathrm{CM}(A))=C \ell(A)$ as shown in Proposition 4.2. In this case $\operatorname{Pic}(\mathrm{CM}(A))$ may be a non-trivial group. Therefore, to complete the proof of Theorem 1.1, it remains to prove the following theorem.

Theorem 6.2. Let A be a Cohen-Macaulay local k-algebra. Suppose that $A$ has only an isolated singularity and $\operatorname{dim} A \geq 3$. Then $\operatorname{Pic}(\mathrm{CM}(A))$ is a trivial group. Hence we have an isomorphism $\operatorname{Aut}_{k}(\mathrm{CM}(A)) \cong \operatorname{Aut}_{k \text {-alg }}(A)$ as groups.

To show this, we shall prove a stronger result.

Theorem 6.3. Let A be a Cohen-Macaulay local k-algebra of any dimension. Suppose that $A$ is regular in codimension two, i.e., $A_{\mathfrak{p}}$ is a regular local ring for any prime ideal $\mathfrak{p}$ with $\mathrm{ht}(\mathfrak{p})=2$. Then $\operatorname{Pic}(\operatorname{CM}(A))$ is a trivial group.

Proof. If $\operatorname{dim} A=0$, then $\mathrm{CM}(A)=A$-mod and hence $\operatorname{Pic}(\mathrm{CM}(A))=$ Pic $A$ is a trivial group by Example 3.8. If $\operatorname{dim} A=1$, then $\operatorname{CM}(A)=d^{\geq 1}(A)$ and we have shown in Proposition 4.4 that $\operatorname{Pic}(\mathrm{CM}(A))$ is again a trivial group. If $\operatorname{dim} A=2$, then our assumption says that $A$ is a regular local ring hence a UFD. Therefore $\operatorname{Pic}(\mathrm{CM}(A)) \cong C \ell(A)$ is a trivial group.

In the rest we may assume $d=\operatorname{dim} A \geq 3$. Let $[M] \in \operatorname{Pic}(\operatorname{CM}(A))$. Assuming that $M$ is not free, we have only to show a contradiction. Take a free cover $F$ of $M$ and we obtain an exact sequence

$$
0 \longrightarrow \Omega(M) \longrightarrow F \longrightarrow M \longrightarrow 0 .
$$

Note that the first syzygy module $\Omega(M)$ is also a maximal Cohen-Macaulay module. Apply $\operatorname{Hom}_{A}(M,-)$ to the sequence, and we get an exact sequence

$0 \longrightarrow \operatorname{Hom}_{A}(M, \Omega(M)) \longrightarrow \operatorname{Hom}_{A}(M, F)$

$$
\longrightarrow \operatorname{Hom}_{A}(M, M) \stackrel{f}{\longrightarrow} \operatorname{Ext}_{A}^{1}(M, \Omega(M)) .
$$

Notice that $f \neq 0$, since we have assumed that $M$ is not free. Because of the assumption, we see that $M_{\mathfrak{p}}$ is a free $A_{\mathfrak{p}}$-module for any prime ideal $\mathfrak{p}$ with ht $(\mathfrak{p})=2$. Thus $\operatorname{Ext}_{A}^{1}(M, \Omega(M))_{\mathfrak{p}}=0$ for those prime ideals $\mathfrak{p}$. This implies that $\operatorname{dim} \operatorname{Ext}_{A}^{1}(M, \Omega(M)) \leq d-3$, hence the image $\operatorname{Im}(f)$ is also 
a nontrivial $A$-module of dimension at most $d-3$. In particular, we have $\operatorname{depth} \operatorname{Im}(f) \leq d-3$.

On the other hand, since $\left.\operatorname{Hom}_{A}(M,-)\right|_{\mathrm{CM}(A)}$ is a functor from $\mathrm{CM}(A)$ to itself, the modules $\operatorname{Hom}_{A}(M, \Omega(M)), \operatorname{Hom}_{A}(M, F)$ and $\operatorname{Hom}_{A}(M, M)$ have depth $d$. Hence we conclude from the depth argument [4, Proposition 1.2.9] that depth $\operatorname{Im}(f) \geq d-2$. This is a contradiction, and the proof is completed.

\section{Remarks on the duality of an additive full subcategory}

Let $A$ be a $k$-algebra and let $\subseteq$ be an additive full subcategory of $A$-Mod. In this section we are interested in the $k$-linear dualities of $\mathfrak{S}$.

Definition 7.1. An additive functor $D: \subseteq 5 \mathfrak{C}^{\mathrm{op}}$ is called a $k$-linear duality of $\mathfrak{S}$ if it is a $k$-linear functor which gives an equivalence between the categories $\mathbb{C}$ and $\mathfrak{C}^{\mathrm{op}}$.

The following is a direct consequence of Theorem 2.7.

LEMMA 7.2. Let $\mathbb{5}$ be an additive full subcategory of A-Mod which contains $A$ as an object, where $A$ is a commutative $k$-algebra. Suppose there is a $k$ linear duality $D: \mathfrak{S} \rightarrow \mathfrak{C}^{\mathrm{op}}$. Then there is an A-module $\omega \in \mathfrak{\complement}$ and a $k$-algebra automorphism $\sigma \in \operatorname{Aut}_{k \text {-alg }}(A)$ such that $\left.D \cong \operatorname{Hom}_{A}\left(-, \omega_{\sigma}\right)\right|_{\Subset}$ as functors on c5.

In particular, every A-linear duality $D$ of $\sqrt{5}$ is of the form $\left.\operatorname{Hom}_{A}(-, \omega)\right|_{\mathfrak{E}}$ for some $\omega \in \mathbb{C}$.

We say that an object $\omega \in \mathfrak{C}$ is a dualizing module of $\mathfrak{\complement}$ if the functor $\operatorname{Hom}_{A}(-, \omega)$ gives an $A$-linear duality of $\mathfrak{5}$.

EXAMPLE 7.3.

(1) If $A$ is a Cohen-Macaulay local ring with canonical module $\omega_{A}$, then $\omega_{A}$ is a dualizing module of the category $\operatorname{CM}(A)$.

(2) Let $A$ be a Krull domain (resp. a Noetherian normal domain). Then the free module $A$ of rank one is a dualizing module of $\operatorname{Ref}(A)$ (resp. $\operatorname{ref}(A)$ ). (See Propositions 4.1 and 4.2.)

(3) Let $(A, \mathfrak{m})$ be a Noetherian complete local ring and let $E$ be the injective envelope of $A / \mathrm{m}$. Then $E$ is a dualizing module of the category $\operatorname{Mat}(A)$. (See Proposition 4.5.)

In all these examples, the duality functor $D$ satisfies $D^{2} \cong 1$. We remark on this property in the following proposition.

Proposition 7.4. Let A be a commutative k-algebra and let $\subseteq$ be an additive full subcategory of $A$-Mod such that $A \in \mathfrak{C}$. Then every A-linear duality $D$ of 
(5 satisfies the equality $D^{2} \cong 1_{\mathfrak{c}}$. In particular, every dualizing module $\omega$ of 5 satisfies $\operatorname{End}_{A}(\omega) \cong A$ as A-modules.

Proof. It follows from Lemma 2.2 that the $k$-algebra homomorphism $\alpha$ : $A \rightarrow \operatorname{End}(D)$ is an isomorphism. On the other hand, since we may write $D=\left.\operatorname{Hom}_{A}(-, \omega)\right|_{\mathfrak{c}}$ for a dualizing module $\omega \in \mathfrak{C}$, Yoneda's lemma forces that $\operatorname{End}(D) \cong \operatorname{End}_{A}(\omega)$ as $A$-modules. Hence we have isomorphisms of A-modules

$$
D^{2}(A) \cong \operatorname{Hom}_{A}\left(\operatorname{Hom}_{A}(A, \omega), \omega\right) \cong \operatorname{End}_{A}(\omega) \cong A .
$$

Since $D^{2}: \mathfrak{C} \rightarrow \mathfrak{C}$ is a covariant equivalence, we can apply Corollary 2.6 to get $D^{2} \cong 1_{\mathfrak{c}}$.

Note that if $\omega$ is a dualizing module of $\subseteq$ and if $[M] \in \operatorname{Pic}(\mathfrak{C})$, then $\operatorname{Hom}_{A}(M, \omega)$ is also a dualizing module of $\mathfrak{E}$. In fact, we have the following isomorphism of functors by the adjoint formula.

$$
\left.\left.\left.\operatorname{Hom}_{A}\left(-, \operatorname{Hom}_{A}(M, \omega)\right)\right|_{\mathfrak{E}} \cong \operatorname{Hom}_{A}(M,-)\right|_{\mathfrak{c}} \circ \operatorname{Hom}_{A}(-, \omega)\right|_{\mathfrak{E}},
$$

where the right hand side is an $A$-linear duality of $\mathbb{C}$.

Now we prove the main theorem of this section.

THEOREM 7.5. Let A be a commutative $k$-algebra, and let $\mathbb{\mathfrak { c }}$ be an additive full subcategory of $A-M o d$ that is stable under $\operatorname{Aut}_{k-\mathrm{alg}}(A)$ and $A \in \mathfrak{C}$. Suppose that $\mathfrak{S}$ admits a dualizing module $\omega$. Let $D$ be an arbitrary $k$-linear duality of $\widetilde{C}$. Then there exist an automorphism $\sigma \in \operatorname{Aut}_{k \text {-alg }}(A)$ and an element $[M] \in \operatorname{Pic}(\mathbb{S})$ such that there is an isomorphism of functors

$$
\left.\left.D \cong \sigma_{*}\right|_{\mathfrak{c}} \circ \operatorname{Hom}_{A}\left(-, \operatorname{Hom}_{A}(M, \omega)\right)\right|_{\mathfrak{c}} .
$$

Proof. In fact, the composition of functors $D \circ\left(\left.\operatorname{Hom}_{A}(-, \omega)\right|_{\mathfrak{S}}\right)^{-1}$ is a $k$-linear automorphism of $\mathfrak{c}$, hence it follows from Lemma 5.8 that

$$
\left.\left.D \circ\left(\left.\operatorname{Hom}_{A}(-, \omega)\right|_{\mathfrak{S}}\right)^{-1} \cong \sigma_{*}\right|_{\mathfrak{c}} \circ \operatorname{Hom}_{A}(M,-)\right|_{\mathfrak{E}},
$$

for some $\sigma \in \operatorname{Aut}_{k \text {-alg }}(A)$ and $[M] \in \operatorname{Pic}(\mathfrak{S})$. Therefore we have

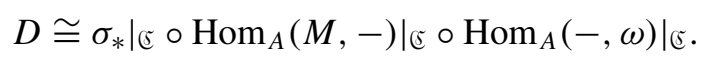

By the isomorphism (2) above, we see that the right hand side is equal to $\left.\left.\sigma_{*}\right|_{\mathfrak{E}} \circ \operatorname{Hom}_{A}\left(-, \operatorname{Hom}_{A}(M, \omega)\right)\right|_{\mathfrak{E}}$. 


\title{
REFERENCES
}

1. Auslander, M., Reiten, I., and Smalø, S., Representation Theory of Artin Algebras, rev. ed., Cambridge Studies in Adv. Math. 36, Cambridge Univ. Press, Cambridge 1997.

2. Bass, H., Algebraic K-theory, Benjamin, New York 1968.

3. Bourbaki, N., Commutative Algebra, Chapters 1-7, Elements of Math. (Berlin), Springer, Berlin 1998.

4. Bruns, W., and Herzog, J., Cohen-Macaulay Rings, rev. ed., Cambridge Studies in Adv. Math. 39, Cambridge Univ. Press, Cambridge 1998.

5. Enochs, E., Flat covers and flat cotorsion modules, Proc. Amer. Math. Soc. 92 (1984), 179184.

6. Fossum, R., The Divisor Class Group of a Krull Domain, Ergebnisse der Math. und ihrer Grenzgebiete 74, Springer, New York 1973.

7. Matlis, E., Injective modules over Noetherian rings, Pacific J. Math. 8 (1958), 511-528.

8. Matsumura, H., Commutative Ring Theory, Cambridge Studies in Adv. Math. 8, Cambridge Univ. Press, Cambridge 1989.

9. Rickard, J., Morita theory for derived categories, J. London Math. Soc. (2) 39 (1989), 436456.

10. Rickard, J., Derived equivalences as derived functors, J. London Math. Soc. (2) 43 (1991), $37-48$.

11. Yekutieli, A., Dualizing complexes, Morita equivalence and the derived Picard group of a ring, J. London Math. Soc. (2) 60 (1999), 723-746.

12. Yoshino, Y., Cohen-Macaulay Modules over Cohen-Macaulay Rings, London Math. Soc. Lecture Note 146, Cambridge Univ. Press, Cambridge 1990.

\author{
DEPARTMENT OF MATHEMATICS \\ OKAYAMA UNIVERSITY \\ 700-8530 OKAYAMA \\ JAPAN \\ E-mail: naoya_h@math.okayama-u.ac.jp \\ E-mail: yoshino@math.okayama-u.ac.jp
}

